\title{
Nanoradar based on nonlinear dimer nanoantenna
}

\author{
Nadezhda Lapshina, ${ }^{1, *}$ Roman Noskov, ${ }^{1}$ and Yuri Kivshar ${ }^{1,2}$ \\ ${ }^{1}$ National Research University of Information Technologies, Mechanics and Optics (ITMO), St. Petersburg 197101, Russia \\ ${ }^{2}$ Nonlinear Physics Center, Research School of Physics and Engineering, Australian National University, Canberra ACT 0200, Australia \\ *Corresponding author: n.lapshina@phoi.ifmo.ru
}

Received July 9, 2012; accepted August 2, 2012;

posted August 10, 2012 (Doc. ID 172250); published September 14, 2012

\begin{abstract}
We introduce the concept of a nanoradar based on the operation of a nonlinear plasmonic nanoantenna. The nanoradar action originates from modulational instability occurring in a dimer nanoantenna consisting of two subwavelength nonlinear nanoparticles. Modulation instability causes a dynamical energy exchange between the nanoantenna eigenmodes resulting in periodic scanning of the nanoantenna scattering pattern. Such nanoradar demonstrates a wide scanning sector, low operation threshold, and ultrafast time response being potentially useful for many applications in nanophotonics circuitry. () 2012 Optical Society of America

OCIS codes: $250.5403,230.6120,190.3100,320.2250$.
\end{abstract}

The study of optical nanoantennas is a rapidly developing area of research [1]. Nanoantennas keep promises for a variety of applications in nanophotonics with advantages of enhanced light-matter interaction. For many applications, an active control over the nanoantenna radiation pattern is required. However, the nanoantenna operation is usually based on one lasing mode that has better directivity and/or higher Purcell factor [2,3]. Nevertheless, for nanoantennas with broken material and/or geometrical symmetry, it was suggested to employ spectral tunability and variable directionality based on switching between different modes $[\underline{4}, 5]$, which also can be realized through nonlinear loads $[\underline{6}-\underline{9}]$.

In this Letter, we suggest a novel way for the dynamic control of the nanoantenna directivity by exploiting modulational instability (MI) of a nonlinear dimer nanoantenna. We demonstrate that the development of MI in a nanodimer composed of two nonlinear silver nanoparticles can lead to a periodic exchange of the power between the eigenmodes and result in a periodic variation of the nanoantenna scattering pattern similar to classical phased-array radar systems [10], as illustrated schematically in Fig. 1.

We consider a nanoantenna created by a pair of nanoparticles placed close to each other and embedded into an $\mathrm{SiO}_{2}$ host medium with a permittivity $\varepsilon_{h}=2.15$. We assume that the nanoparticles are equivalent, and they have the radius $a=10 \mathrm{~nm}$ with the center-to-center spacing of $d=30 \mathrm{~nm}$ (see the insert in Fig. 1). Since the condition $a / d \leq 1 / 3$ is satisfied, we can employ the dipole approximation [11]. Assuming the nanoparticles made of silver with a nonlinear Kerr-like response, we take the dielectric constant in the form $\varepsilon_{\mathrm{Ag}}^{\mathrm{NL}}=\varepsilon_{\mathrm{Ag}}^{L}+$ $\chi^{(3)}\left|\mathbf{E}_{1,2}^{(i n)}\right|^{2}$, where the linear part is given by the Drude formula, $\varepsilon_{\mathrm{Ag}}^{L}=\varepsilon_{\infty}-\omega_{p}^{2} /[\omega(\omega-i \nu)]$, with $\varepsilon_{\infty}=4.96$, $\hbar \omega_{p}=9.54 \mathrm{eV}$, and $\hbar \nu=0.055 \mathrm{eV}[\underline{12}]$, and $\mathbf{E}_{1,2}^{(\mathrm{in})}$ are the local fields inside the first and second particles. For spherical silver nanoparticles with the radius $10 \mathrm{~nm}$, we use the nonlinear coefficient with the value $\chi^{(3)} \simeq 3 \times 10^{-9}$ esu from [13], which is much larger than the cubic nonlinearity of $\mathrm{SiO}_{2}\left(\sim 10^{-15}\right.$ esu [14] $)$, the latter being neglected. Our aim is to study the temporal evolution of the scattering pattern of this dimer nanoantenna in the nonlinear regime when the response of this structure undergoes MI.

We study a nanoparticle dimer driven by a plane wave (see the insert in Fig. 1) with the frequency close to the frequency of the surface plasmon resonance of an individual particle, and analyze the dynamical response of the particles through the evolution of their polarizations, $\mathbf{p}_{1,2}$. To derive the corresponding nonlinear equations for the polarizations, we employ the model recently applied for the study of strongly localized modes in a chain of nanoparticles [15]. In the case when the external electric field $E^{(\mathrm{ex})}$ is polarized along the dimer axis, this model yields the following system of coupled equations for the slowly varying amplitudes of the particle dipole moments:

$$
\begin{aligned}
& -i \frac{d P_{1}^{\|}}{d \tau}+\left(\Omega-i \gamma+\left|P_{1}^{\|}\right|^{2}\right) P_{1}^{\|}+G^{\|} P_{2}^{\|}=E, \\
& -i \frac{d P_{2}^{\|}}{d \tau}+\left(\Omega-i \gamma+\left|P_{2}^{\|}\right|^{2}\right) P_{2}^{\|}+G^{\|} P_{1}^{\|}=E,
\end{aligned}
$$

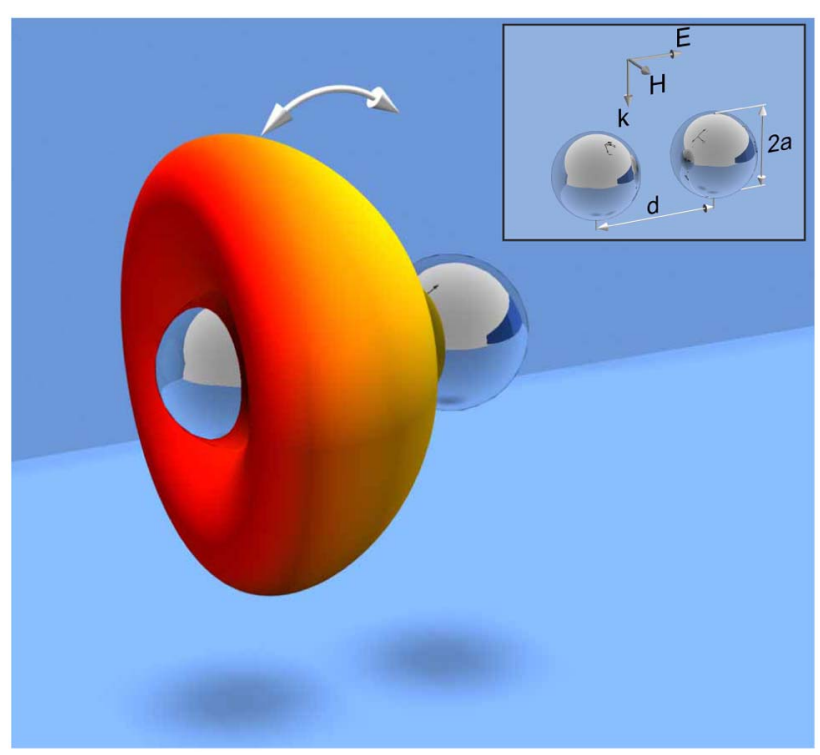

Fig. 1. (Color online) Schematic of the scattering pattern of a dimer nanoantenna. Insert shows the structure, parameters, and direction of an incident plane wave. 
where the effective coefficient $G^{\|}=3 \varepsilon_{h} /\left(\varepsilon_{\infty}+2 \varepsilon_{h}\right)$ $(a / d)^{3}\left(i k_{0} d+1\right) \exp \left(-i k_{0} d\right)$ describes the strength of the particle interaction, and we use the dimensionless functions

$$
P_{1,2}^{\|}=\frac{\sqrt{\chi^{(3)}} p_{1,2}^{\|}}{\sqrt{2\left(\varepsilon_{\infty}+2 \varepsilon_{h}\right)} \varepsilon_{h} a^{3}},
$$

and $E=-3 \varepsilon_{h}\left[\chi^{(3)}\right]^{1 / 2} E^{(\mathrm{ex})} /\left[2\left(\varepsilon_{\infty}+2 \varepsilon_{h}\right)\right]^{3 / 2}$ for the slowly varying amplitudes of the particle dipole moments and external electric field, respectively. The index ' $\|$ ' stands for the longitudinal components with respect to the dimer axis, the parameter $\gamma=\nu /\left(2 \omega_{0}\right)+\left(k_{0} a\right)^{3} \varepsilon_{h} /$ $\left(\varepsilon_{\infty}+2 \varepsilon_{h}\right)$ describes thermal and radiation losses of particles, $\omega_{0}=\omega_{p} /\left(\varepsilon_{\infty}+2 \varepsilon_{h}\right)^{1 / 2}$ is the frequency of the surface plasmon resonance of an individual particle, $k_{0}=\omega_{0} / c \sqrt{\varepsilon_{h}}, \Omega=\left(\omega-\omega_{0}\right) / \omega_{0}$, and $\tau=\omega_{0} t$. For the chosen configuration, $\hbar \omega_{0}=3.14 \mathrm{eV}$. Nonlinear terms appeared after expressing $E_{1.2}^{(\mathrm{in})}$ via $p_{1.2}^{\|}$[ $[\underline{15}]$. Equation (1) describes the temporal nonlinear dynamics of a metallic nanodimer driven by a plane wave with the frequency $\omega \sim \omega_{0}$.

The stationary states of this system are described by homogeneous solutions when the particle dipole moments coincide, $P_{1,2}^{\|}=P_{0}^{\|}$; they can be found as solutions of the nonlinear equation,

$$
\left(-i \gamma+\Omega+G^{\|}+\left|P_{0}^{\|}\right|^{2}\right) P_{0}^{\|}=E_{0},
$$

where $E_{0}$ is the stationary amplitude of the external electric field. This equation has multiple solutions for $\Omega<-\operatorname{Re} G^{\|}-\sqrt{3}\left(\gamma-\operatorname{Im} G^{\|}\right)$, so that the system of two nonlinear nanoparticles is expected to have a bistable regime.

Next, we analyze linear stability of the homogeneous stationary states with respect to weak perturbations taken in the form of an asymmetric longitudinal eigenmode. By applying a standard technique [16], we derive the expression for the instability growth rate,

$$
\lambda=-\operatorname{Im} G^{\|}-\gamma+\left\{\left|P_{0}^{\|}\right|^{4}-\left(2\left|P_{0}^{\|}\right|^{2}+\Omega-\operatorname{Re} G^{\|}\right)^{2}\right\}^{1 / 2} .
$$

The homogenous state (2) becomes unstable provided $\lambda\left(\Omega, E_{0}^{2}\right)>0$, and the condition $\lambda=0$ defines the boundary of the MI region on the plane $\left(\Omega, E_{0}^{2}\right)$, as shown in Fig. 2. Remarkably, MI occurs for the whole upper branch in the bistable region, and it extends much further (see the inset in Fig. 2).

To study the evolution of this nonlinear system after the onset of instability, we perform numerical simulations of Eq. (1) at zero initial conditions and fixed driving frequency $\Omega=-0.12(\hbar \omega \cong 2.76 \mathrm{eV})$, when the external field $E$ grows slowly approaching the threshold value $E_{\text {sat }}=0.014$ corresponding to the region of MI. The results are presented in Figs. 3(a) and 3(b), where we observe that instability results in the spontaneous excitation of an asymmetric eigenmode of the dimer leading to an energy exchange between the symmetric and asymmetric eigenmodes. For weak external fields, the particle polarizations coincide being described by the phase-space trajectories corresponding to the focus.

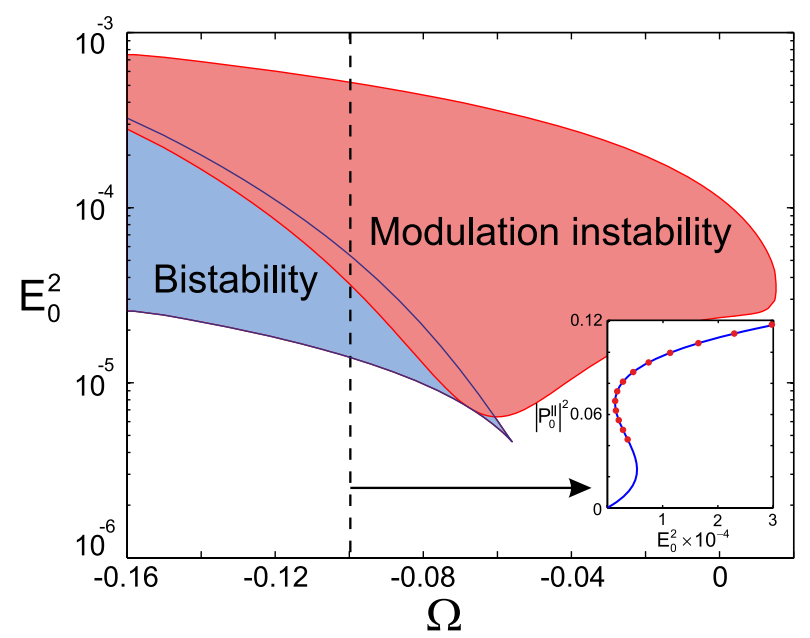

Fig. 2. (Color online) Bifurcation diagram on the parameter plane of $E_{0}^{2}$ and $\Omega=\left(\omega-\omega_{0}\right) / \omega_{0}$, with the regions of bistability and modulation instability. Inset: dependence of the polarization $\left|P_{0}^{\|}\right|^{2}$ on $E_{0}^{2}$ at $\Omega=-0.1$ (along the dashed vertical line on the main plot). Red dots mark the region of modulation instability.

When $E$ grows reaching the region of MI, this state becomes unstable, and the phase trajectories get attracted by two different limiting circles, as shown in Fig. 3(b).

MI breaks the phase locking between the particle dipole moments and the external field. Consequently, self-modulation dynamical response of a nanodimer results in temporal modulation of the nanoantenna scattering cross section and rotation of the scattering pattern, as shown in Figs. 4(a)-4(e). The period of self-oscillations and the contrast of scattering are $90 \mathrm{fs}$ and 18, respectively. This modulation is much faster than that typically observed using plasmonic antennas with nonlinear semiconductor loading [].

We notice a relatively wide angular scanning sector $\Delta \theta_{m l} \simeq 50^{\circ}$ of the nanodimer's radiation pattern. However, using a nanoradar in practice may require much better directivity than the directivity of a dimer antenna, for which one may utilize, e.g., a high-permittivity dielectric sphere as a director [2].

The saturation amplitude of the external field $E_{\text {sat }}=$ 0.014 corresponds to the intensity of $21.6 \mathrm{MW} / \mathrm{cm}^{2}$. Such a high illuminating power can lead to thermal damage
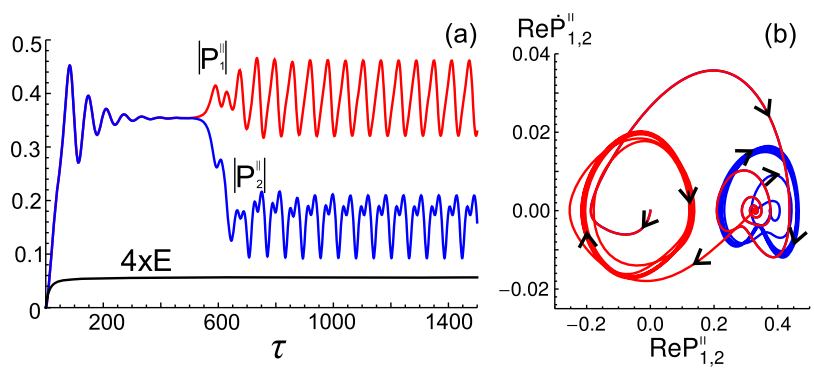

Fig. 3. (Color online) (a) Temporal evolution of the polarizations $\left|P_{1}^{\|}\right|$(red) and $\left|P_{2}^{\|}\right|$(blue), when the external field $E$ (black) approaches and crosses the instability threshold. Instability leads to an energy exchange between symmetric and asymmetric eigenmodes. (b) Phase trajectories associated with the dynamics shown in (a). 

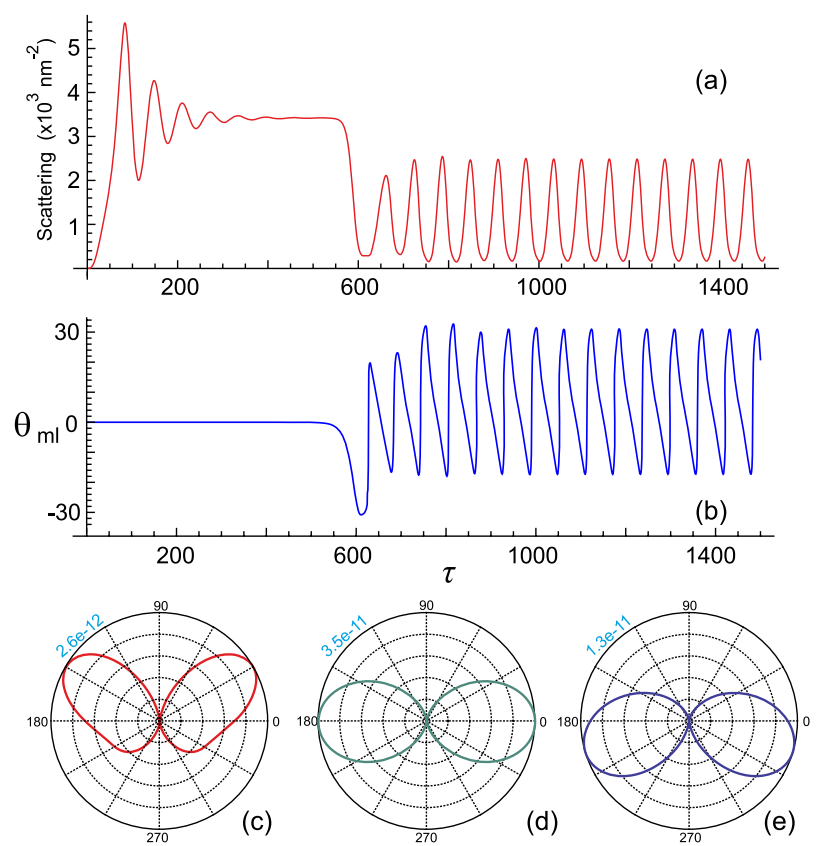

Fig. 4. (Color online) Temporal evolution of (a) scattering cross section and (b) oscillation angle $\theta_{m l}$ corresponding to the major scattering lobe. (c)-(e) Snapshots of the scattering intensities (in watts) corresponding to maximal deviations from the initial state (red and blue) and an intermediate position (green).

unless we operate with short pulses. To estimate the maximal pulse duration, we use the value of the ablation threshold of $3.96 \mathrm{~J} / \mathrm{cm}^{2}$ obtained for silver particles in an $\mathrm{SiO}_{2}$ host matrix in the picosecond regime of illumination [17]. Taking into account the amplification of the electric field inside the Ag nanoparticle due to surface plasmon resonance and the required intensity, we evaluate the maximal pulse duration as $0.5 \mathrm{~ns}$, which is much longer than the characteristic period of self-oscillations. Thus, the predicted scattering pattern rotation can be readily observed in experiment.

In conclusion, we have suggested a novel approach for realizing an active control over the optical nanoantenna directivity. We have shown that MI in a nonlinear plasmonic dimer can lead to a dynamical energy exchange between the nanoantenna eigenmodes resulting in a periodical variation of the nanoantenna scattering pattern. Such actively tunable nanoantennas may operate in a wide scanning sector with a low operation threshold and ultrafast modulation. Further optimization may be possible by considering other designs of nanoantennas with higher directionality, such as Yagi-Uda [1] or metaldielectric [2] configurations. Another possibility for tunability of the nanoradar is a variation in the plane wave incidence, intensity, and frequency. In this case, different scenarios of the development of MI include transitions to other steady states, bifurcations, period doubling, and even a transition to chaos. We have studied some of such regimes, and results will be published elsewhere.

The authors thank P. Belov and A. Zharov for useful discussions, and they acknowledge a support from the Ministry of Education and Science of the Russian Federation and the Australian Research Council.

\section{References}

1. P. Biagioni, J. Huang, and B. Hecht, Rep. Prog. Phys. 75, 024402 (2012).

2. A. Devilez, B. Stout, and N. Bonod, ACS Nano 4, 3390 (2010).

3. A. E. Krasnok, A. E. Miroshnichenko, P. A. Belov, and Yu. S. Kivshar, JETP Lett. 94, 593 (2011).

4. N. Bonod, A. Devilez, B. Rolly, S. Bidault, and B. Stout, Phys. Rev. B 82, 115429 (2010).

5. T. Shegai, S. Chen, V. Miljković, G. Zengin, P. Johansson, and M. Käll, Nat. Commun. 2, 481 (2011).

6. P.-Y. Chen and A. Alu, Phys. Rev. B 82, 235405 (2010).

7. N. Large, M. Abb, J. Aizpurua, and O. L. Muskens, Nano Lett. 10, 1741 (2010).

8. M. Abb, P. Albella, J. Aizpurua, and O. L. Muskens, Nano Lett. 11, 2457 (2011).

9. I. S. Maksymov, A. E. Miroshnichenko, and Yu. S. Kivshar, Opt. Express 20, 8929 (2012).

10. T. Jeffrey, Phased-Array Radar Design (SciTech Publishing, 2009).

11. I. Romero, J. Aizpurua, G. W. Bryant, and F. J. García de Abajo, Opt. Express 14, 9988 (2006).

12. P. B. Johnson and R. W. Christy, Phys. Rev. B 6, 4370 (1972).

13. V. P. Drachev, A. K. Buin, H. Nakotte, and V. M. Shalaev, Nano Lett. 4, 1535 (2004).

14. M. J. Weber, Handbook of Optical Materials (CRC, 2003).

15. R. E. Noskov, P. A. Belov, and Yu. S. Kivshar, Phys. Rev. Lett. 108, 093901 (2012).

16. M. I. Rabinovich and D. I. Trubetskov, Oscillations and Waves in Linear and Nonlinear Systems (Kluwer, 1989).

17. C. Torres-Torres, N. Peréa-López, J. A. Reyes-Esqueda, L. Rodríguez-Fernández, A. Crespo-Sosa, J. C. Cheang-Wong, and A. Oliver, Int. J. Nanomedicine 5, 925 (2010). 